\title{
The Histological Changes and Caspase-3 Expression during Wound Healing in Contused Skeletal Muscle in Male Albino Rats
}

\author{
Irene Atef Fawzy ,Morid Malak Hanna', and Nashwa Fathy El-Tahawy²
}

\author{
${ }^{1}$ Forensic Medicine \& Toxicology Department \\ ${ }^{2}$ Histology Department \\ Faculty of Medicine, Minia University, Minia, Egypt
}

\begin{abstract}
Wound age estimation is one of the most critical issues for forensic pathologists. This study aimed to assess the time dependant histological changes versus the expression of Caspase 3 histochemically during contused skeletal muscle healing to discuss the practical suitability of the latter as a parameter for wound age determination in the living instead of histological change. Forty -six male albino rats were used in this study and divided into five groups, control group and four contused groups. At 12 hours, 3, 5 and 7 days after contusion, rats were killed and muscle samples were dissected from right hind limb of all rats. Histological examination was made and changes of Caspase- 3 activity were assessed by imunohistochemistry, the results showed normal morphology of striated muscles and no Caspase-3 was detected in skeletal muscle in the control group. Light microscopic examination of contused groups revealed that polymorphonulcear cells (PMNs) were detected at 12 hours post contusion. Degenerated skeletal muscle fibers were phagocytosed gradually at 3 days, while most of degenerated fibers were phagocytosed and disappeared at 5 days post injury. From 3 days post-injury, fibroblast cells were present in the wound zones, and multinucleated myotubes augmented remarkably at 5-7 days. The expression level of Caspase- 3 in the peripheral area of contused rat skeletal muscle increased gradually and maximized on the fifth day after injury, and then decreased gradually. Caspase- 3 was also detected in the regenerated skeletal muscle within 7 days after injury. In conclusion, the expression of Capase-3 in the peripheral area of contused rat skeletal muscle indicated that it may play a pivotal role in apoptosis of the wounded skeletal muscle and may be used as a marker for wound age estimation in the living human being instead of histological changes.
\end{abstract}

\section{Introduction}

$\mathrm{W}$ ound age estimation is one of the most critical issues for forensic pathologists (Stephenson and Bialas, 1996; Sato and Ohshima, 2000; Takamiya et al., 2005). In cases of suspected non accidental injury, it is often necessary to give an opinion on the age of soft-tissue injuries. Although it has been mentioned that wound age can be estimated by observing skin color changes, there is no general consensus on the duration of coloration at each stage and the exact sequence of color change(Sun et al.,2010).

When skeletal muscle is damaged, the tissue repair process starts immediately and is a complicated but well-organized biological phenomenon concomitant with polymorphonulcear cells (PMNs) and round-shaped mononuclear cells (MNCs) infiltration-macrophages, spindle-shaped fibroblastic cells (FBCs) proliferation, composed of three different phases, destruction, repair, and remodeling ( $\mathrm{Yu}$ et al.,2010). Inflammatory cells including polymorphonuclear cells (PMNs), mononuclear cells (MNCs) and fibroblastic cells (FBCs) play important roles during skin wound healing. Apoptosis, a ubiquitous way of cell death in both physiological and pathological conditions, is a vital component in that it is involved in the removal of inflammatory cells and the evolution of granulation tissue into the scar tissue during skin wound healing (Zhao et al., 2009).

Dysregulation of apoptosis during wound healing can lead to pathologic forms of healing such as excessive scarring and fibrosis. In the process of apoptosis, the factors in apoptotic pathway may play pivotal roles. Most of the studies regarding wound age estimation in skin were conducted by 
immunohistochemical and molecular biological techniques. The expression of chemokines such as interleukin 8 (IL-8), monocyte chemoattractant protein 1 (MCP-1) and macrophage inflammatory protein 1 alpha (MIP-1alpha) by immunohistochemistry were time-dependent in human skin wounds (Kondo et al., 1999). In situ end labeling of DNA fragments (ISEL) was also used to investigate the time-dependent appearance of signs of cell death (Betz et al., 1997). Caspases-3, a member of the cysteine-aspartic acid protease (Caspase) family, plays critical roles in the execution of apoptotic pathway (Li et al., 2011). Studies of experimental models suggested that activated Caspase-3 is a reliable indicator of apoptotic rate (Jeruc et al., 2006)

A Previous study examined the expression of the cannabinoid receptor type 2 (CB2R) by immunohistochemistry, during wound healing contused skeletal muscle in rats (Zhao et al., 2009), While, In the present study, we examined the histopathological changes and the time dependant expression of Caspase 3 immunohistochemically during skeletal muscle wound healing, to discuss their practical suitability as parameters for wound age determination in the living human being.

\section{Materials and Methods}

A total of 46 male albino rats, around 12 to 14 weeks old, weighing between 180 and 250 gm were used in this study. The experimental procedure was conducted in accordance with the guide of the care and use of laboratory animals approved by the committee of Minia University. The rats were divided into five groups, a control group ( $\mathrm{n}=6)$ and 4 contusion groups (no=10 each). Animals were kept with free access to food and water adlibitum. The rats of the contusion groups were undergone anesthesia with ethyl ether. The right hind limb was shaved, subsequently, the rats were placed on experimental table in a prone position and a 250 -g counterpoise was raised and allowed to fall freely by the effect of gravity, from $150 \mathrm{~cm}$ onto the right hind limb of rats (McBrier et al., 2007). Twelve hours, 3, 5 and 7 days after contusion, rats were sacrificed by decapitation. Approximate $100 \mathrm{mg}$ muscle sample was dissected from the right hind limb of all groups. Then, samples were prepared for histological, pathological examination by light microscope and for the assessment of changes of Caspase- 3 activity by imunohistochemistry.

\section{Histological and immunohistochemical staining}

The obtained skeletal muscles of control and contused groups were fixed in $10 \%$ buffered formalin, followed by paraffin embedding using routine procedures (Zhao et al., 2009). Sections of seven-micrometre were stained with haematoxylin and eosin (H\&E) for histological, pathological examination using a light microscope.

\section{Immunohistochemistry}

A goat polyclonal antibody against activated Caspase-3 (Sigma Aldrich) was used for detection of apoptosis.
Briefly, other seven-micrometre sections were deparaffinized, hydrated then washed in $0.1 \mathrm{M}$ phosphate buffer saline (PBS). Endogenous peroxidases were quenched by treatment with $\mathrm{H}_{2} \mathrm{O}_{2}$ in methanol (Peroxidase blocking solution) followed by washing in tris buffer saline (TBS). Non-specific binding of $\mathrm{IgG}$ was blocked using normal goat serum, diluted $1: 50$ in $0.1 \%$ bovine serum albumin with TBS for 30 minutes. Then sections were incubated with the primary rabbit anti-caspase 3 antibody overnight at $4 \circ \mathrm{C}$. Primary $\mathrm{Ab}$ binding was detected using a horseradish peroxidase-conjugated goat anti-rabbit antibody (Vector Laboratories, Burlingame, CA,USA) and visualized by development with 3,3diaminobenzidine (DAB; Sigma). All sections were counterstained with Haematoxylin (Goldspink et al., 2004).

\section{Image capture}

Tissue sections were examined and images were digitally captured using a hardware consisting of a highresolution color digital camera mounted on an Olympus microscope and connected to a computer, then dealt with using adobe Photoshop.

\section{Quantitative analysis}

For quantitative analysis, an area including 500 muscle fibers was defined and the number of immunostained muscle fibers determined as well as the respective incidences and percentages referring to all 500 muscle fibers calculated. All data was presented as mean \pm SD.

\section{Statistical analyses}

All data are presented as means \pm SD. The one-way ANOVA test followed by post-hoc test was used for data analysis between two groups. Difference associated with $\mathrm{P}<0.05$ was considered statistically significant.

\section{Results}

\section{Haematoxylin and eosin results}

In sections stained with hematoxylin and eosin, the control group showed normal morphology of the striated muscles. The muscle fibers are long, with acidophilic striated cytoplasm and multiple flat peripheral nuclei. The myocytes were arranged in bundles which surrounded by the myomisium (fig. 1). Hemorrhage, edema, and degeneration were present in contused skeletal muscle, and a few PMNs appeared at wound zones at 12 hours post-wounding (fig. 2). Degenerated skeletal muscle fibers were phagocytosed gradually, and a large number of PMNs and MNCs accumulated in the wounds at 3 days aged wound (fig. 3 ). MNCs remained abundant at 5 days after wounding while most of degenerated fibers were phagocytosed and disappeared (fig. 4). From 3 days post-injury onward, fibroblast cells (FBCs) were present in the wound zones, and multinucleated myotubes augmented remarkably at 5-7 days where regeneration start to occur (fig. 5).

\section{Immunohistochemical results}

In the uninjured controls, immunoreactivity of normal myofibers was not detected. In the contusion groups, a 
positive staining for caspase-3 were observed in wounded zones especially in 3 and 5 days post-injury groups. In the 7 days post-injury group, sections showed decreased positive reactions due to absorption of most of cells (fig.6).

\section{Morphometric results}

No Caspase-3 was detected in skeletal muscle in the control group. The expression level of Caspase- 3 in the peripheral area of contused rat skeletal muscle increased gradually and maximized on the fifth day after injury, and then decreased gradually but Caspase3 was also detected in the regenerated skeletal muscle 7 days after injury (Table 1 ).

Table 1: One way ANOVA analysis followed by post-hoc test for Caspase-3 positive cells at different time points in control and contused rat skeletal muscles.

\begin{tabular}{|l|c|c|}
\hline \multicolumn{1}{|c|}{ Groups } & Expression level of Caspase-3 (means \pm SE) & P Value \\
\hline $\begin{array}{l}\text { Control } \\
\text { ( } \mathrm{n}=6 \text { rats) }\end{array}$ & $2.31 \pm 0.79$ & \\
\hline $\begin{array}{l}12 \text { hours post injury } \\
\text { ( }=10 \text { rats) }\end{array}$ & $18.91 \pm 5.49$ & $0.06^{*}$ \\
\hline $\begin{array}{l}\text { 3days post injury } \\
\text { (n=10 rats) }\end{array}$ & $41.33 \pm 3.82$ & $0.001^{*}$ \\
\hline $\begin{array}{l}\text { 5days post injury } \\
\text { (n=10 rats) }\end{array}$ & $51.28 \pm 3.79$ & $0.001^{*}$ \\
\hline $\begin{array}{l}\text { 7days post injury } \\
\text { (n=10 rats) }\end{array}$ & $34.29 \pm 2.07$ & $0.009^{*}$ \\
\hline $\mathrm{F}$ & 13476.11 & $0.001^{*}$ \\
\hline
\end{tabular}

*Statistic significance over previous interval; $P<0.05$ is significant; $n:$ number; $F$ : Variance ratio of ANOVA test
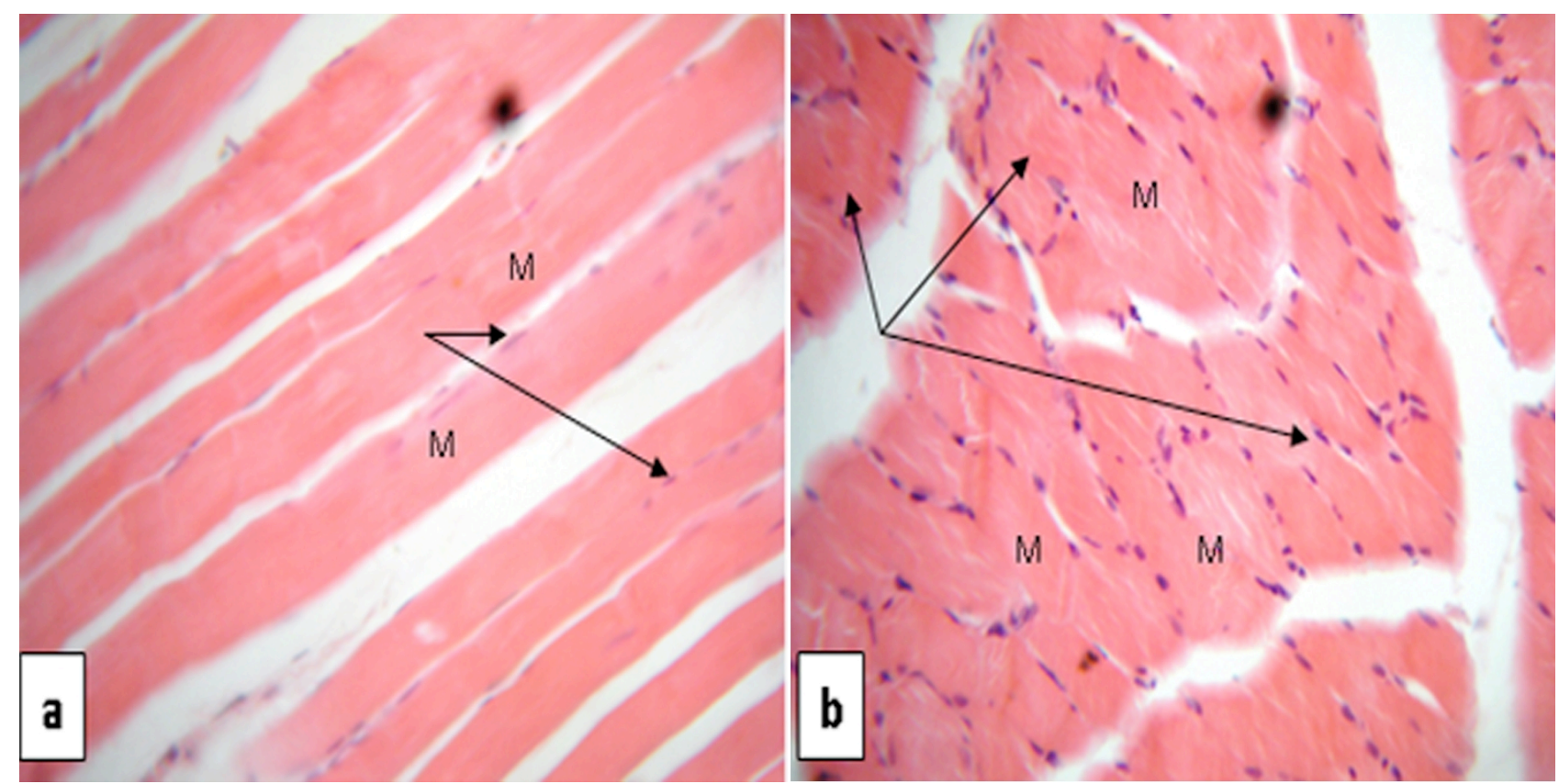

Fig. 1: Photomicrographs of normal rat skeletal muscles showing: a) longitudinal section of skeletal muscle fibers (M) run parallel to each other, the cytoplasm is acidophilic and striated, and the nuclei are flat, multiple and peripheral in position (arrows). b) cross section showing the multinucleated myocytes (M) arranged bundles (arrows). 


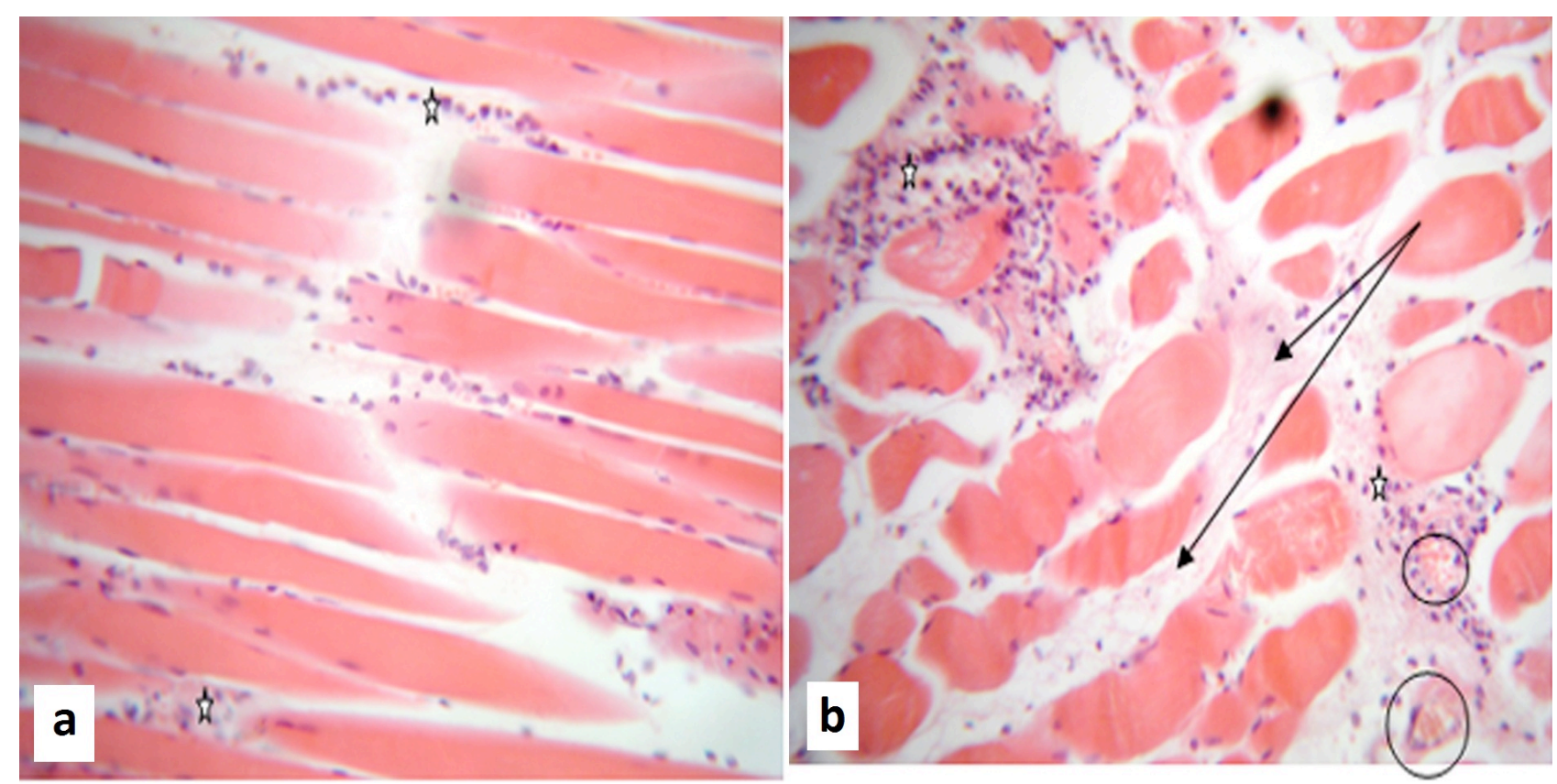

Fig. 2: Photomicrographs of contused rat skeletal muscles 12 hours post injury at the site of contusion in both longitudinal (a) and cross sections (b) showing: inflammatory cell infiltration (stars), edema (arrows), and congestion (circles) around the injured myofibers.

H\&E x40

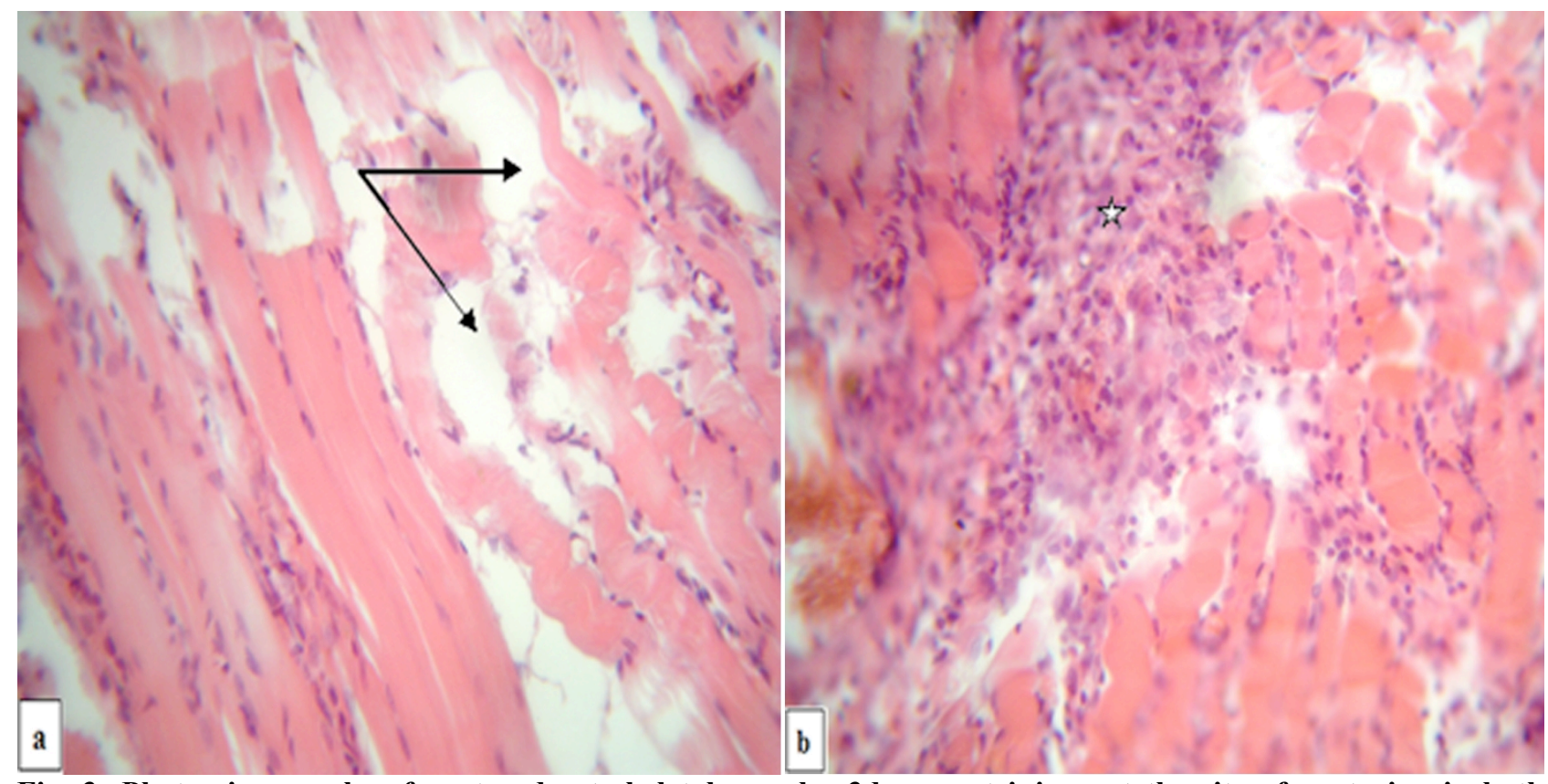

Fig. 3: Photomicrographs of contused rat skeletal muscles 3days post injury at the site of contusion in both longitudinal (a) and cross sections (b) showing: increased inflammatory cell infiltration (stars), injured myofibers started to be phagocytosed leaving bare areas (circles). 


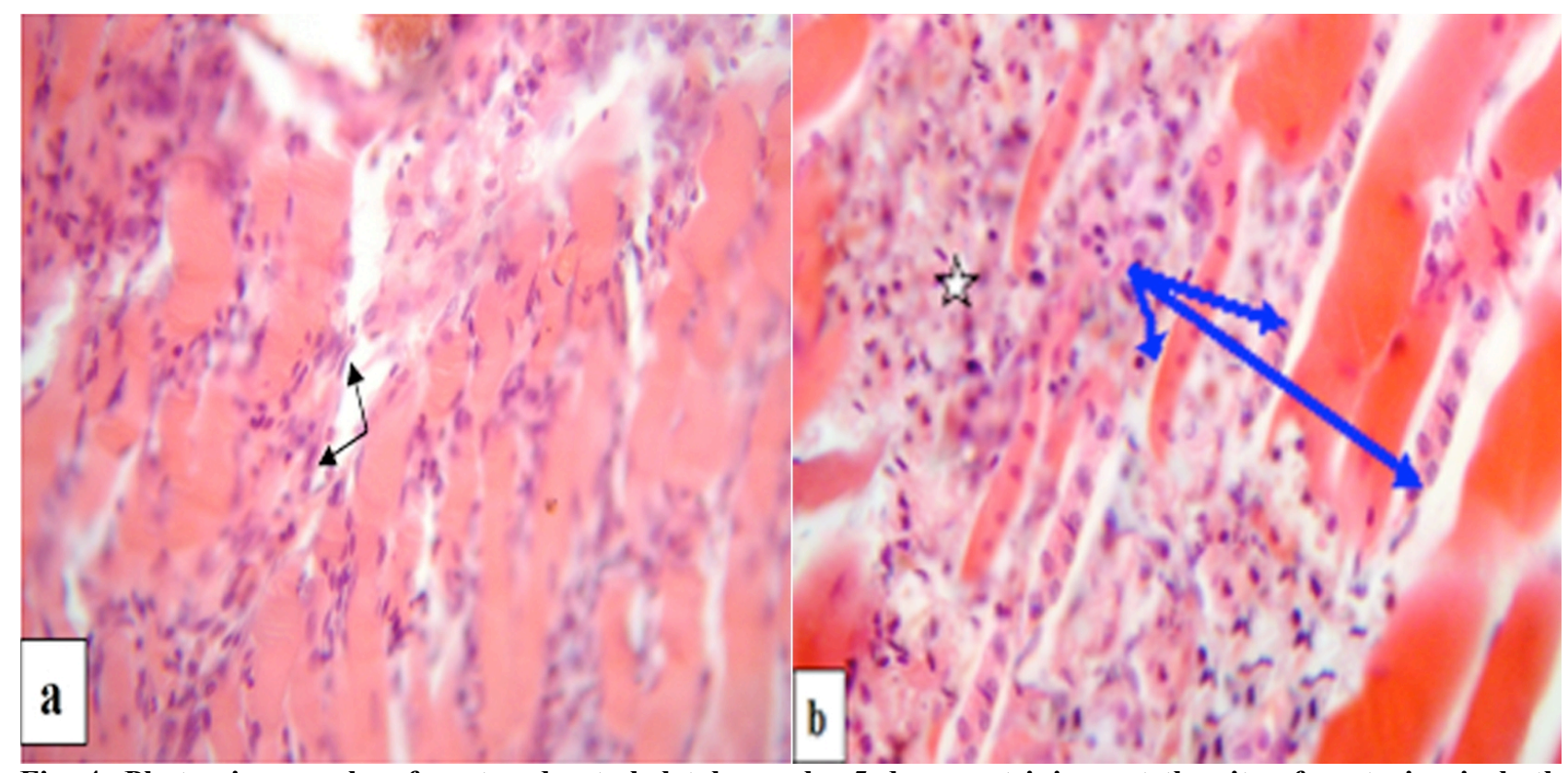

Fig. 4: Photomicrographs of contused rat skeletal muscles 5 days post injury at the site of contusion in both longitudinal (a) and cross sections (b) showing: increased inflammatory cell infiltration (stars), fibroblast cells (arrows), and appearance of myotubes (blue arrows).

H\&E x40

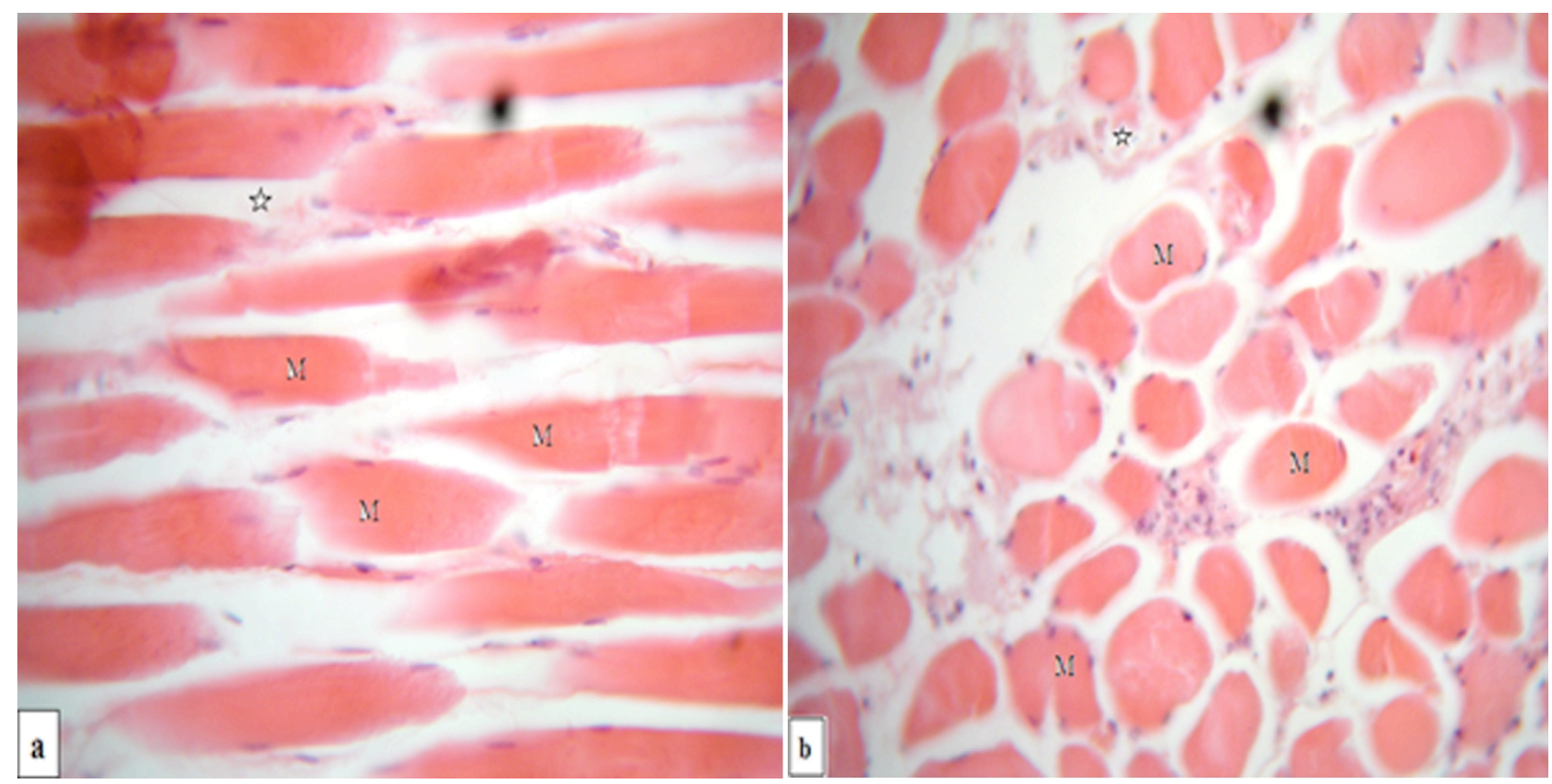

Fig. 5: Photomicrographs of contused rat skeletal muscles 7 days post injury at the site of contusion in both longitudinal (a) and cross sections (b) showing: decreased inflammatory cell infiltration (stars) among regenerated myocytes $(M)$. 


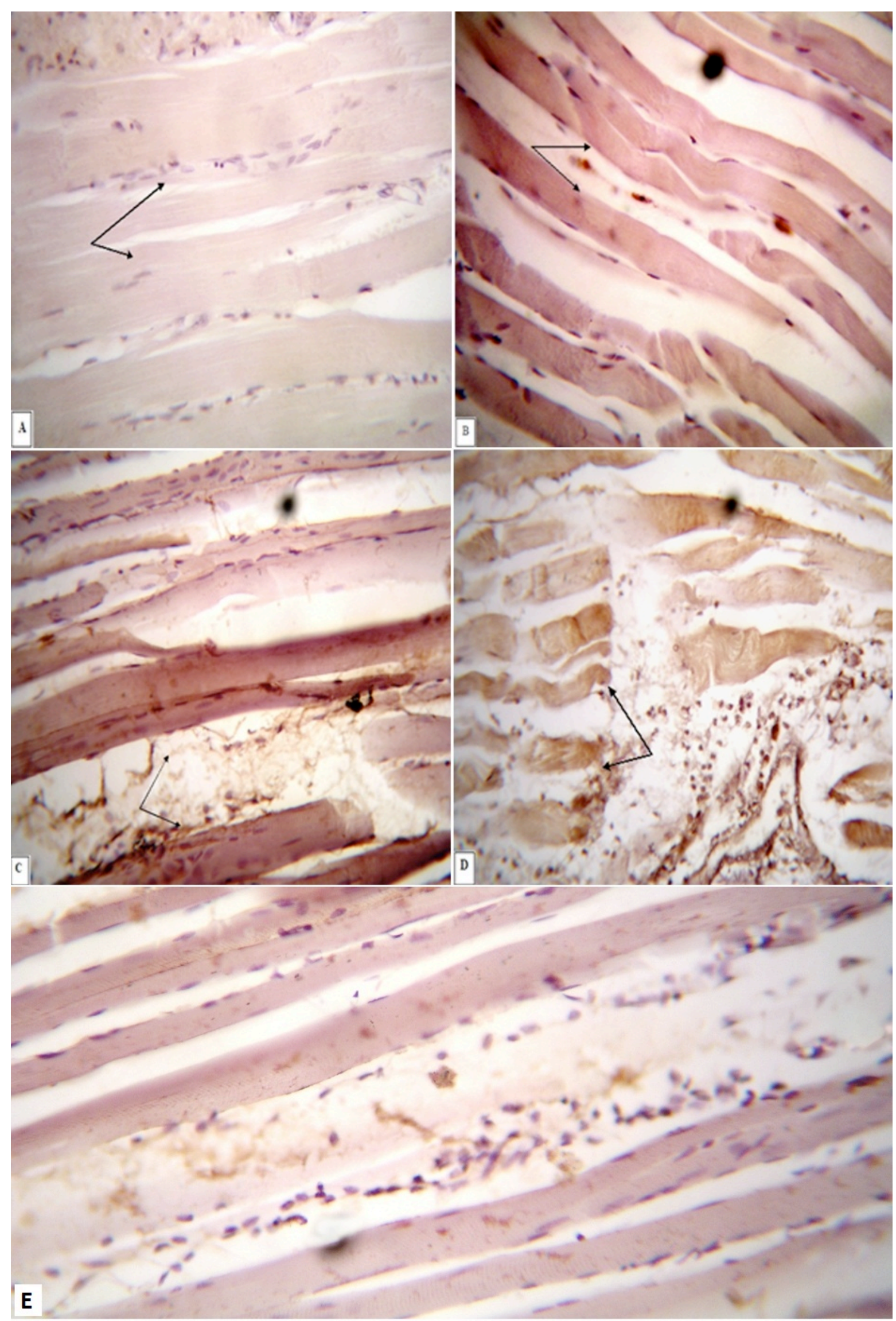

Fig.6: Rat skeletal muscles labeled for activated caspase-3 in: a) control group showing no positive reaction. b) 12 hours after contusion showing positive muscle cells (arrows) at site of contusion. c) 3 days after contusion showing positive darkly stained muscle cells (arrows) at site of contusion. d) 5 days after contusion showing absorption of most of muscle cells with only remnant positive parts (arrows) at site of contusion. e) 7 days after contusion showing positive muscle cells (arrows) at site of contusion.

Immunohistochemistry, counterstained with $\mathrm{H}$ x40 


\section{Discussion}

Wound age estimation is a critical issue which provides indispensable information for the reconstruction of crime scenes and judges the relationship between wounds and cause of death (Kondo, 2007; Ishida et al., 2008; Takamiya et al., 2008).

Wound healing is a complex but spatially and temporally controlled biological process. Generally, it is divided into three phases: inflammatory, proliferative, and maturation (Kondo et al., 2002. Immunohistochemical assays have been used to detect the expression of many biological markers at different time points after injury (Kondo et al., 1999; Dressler et al., 2000; Grellner et al., 2000; Ortmann et al., 2000; Ortiz-Rey et al., 2003; Hayashi et al., 2004; Zhang et al., 2004).

Most researches on forensic wound age determination have been focused on human and murine skin wound healing process in which a variety of biological substances are involved. However, forensic wound age estimation for mammalian skeletal muscle has not been given enough attention yet (Guan et al.,2000;Takamiya et al.,2003; Kagawa et al.,2009; Zhao et al.,2009).

There are many studies on the ultrastructure and pathophysiological development of skeletal muscle contusion healing using a mechanical blunt trauma model in rodents (Hurme et al., 1991; Huard et al., 2002; Wright-Carpenter et al., 2004). Treatment of muscle injuries by local administration of autologous and it is considered that the experimental model mimicked the damage-repair process in human skeletal muscle with a high degree of accuracy and objectivity. Thus, the results obtained from rats in this work are seemingly applicable to the age determination of skeletal muscle contusion in forensic practices.

In this study, we chose the post traumatic intervals starting from 12hours as immunohistochemical staining was restricted for the estimation of wound age less than 8 hours (Sun et al.,2010).

In the present study, hemorrhage, edema, and degeneration were present in contused skeletal muscle, and a few PMNs appeared at wound zones after 12 hours post-traumatic. Degenerated skeletal muscle fibers were phagocytosed gradually, and a large number of PMNs and MNCs accumulated in the wounds of 3 days. Mononuclear cells (MNCs) remained abundant at 5 days after wounding while most of degenerated fibers were phagocytosed and disappeared. From the 3rd day post-injury onward, fibroblast cells were present in the wound zones, and multinucleated myotubes augmented remarkably after 5-7 days where regeneration start to occur. These results are in accordance with Srikuea et al.,(2010) and $\mathrm{Yu}$ et al.,(2010) who found disintegration of the damaged myofibres within $12 \mathrm{~h}$ and newly formed myofibres appeared by day 7 .

It is generally acknowledged that muscle damage induces massive macrophages infiltration into injured zones (McLennan, 1996) and by $24 \mathrm{~h}$, macrophages are the predominant cell type
(Papadimitriou et al., 1990). In vitro, studies have shown that factors produced by injured skeletal muscle such as fibroblast growth factor and platelet derived growth factor are highly chemoattractant for both neutrophils and macrophages, and this activity is apparent within muscle tissue $3 \mathrm{~h}$ post injury and is enhanced by $12 \mathrm{~h}$ (Robertson et al.,1993). Recent studies confirmed that myogenic precursor cells are also chemotactic for macrophages (Chazaud et al., 2009).

Caspase- 3 is involved in myofiber cell death (Sandri et al., 2001). Caspase-3 is activated during apoptosis and as it is known to all, is considered to be the most potential executioner Caspase. Caspase- 3 is cleaved with increase in activity during skin wound healing (Zhao et al., 2009). In this work, the expression level of caspase- 3 in the peripheral area of contused rat skeletal muscle increased gradually and maximized on the fifth day after injury, and then decreased gradually but was also detected in the regenerated skeletal muscle at 7 days after injury. In the same line, Wang et al., (2008) revealed that, the expression level of caspase- 3 in the peripheral area of contused rat skeletal muscle increased gradually and maximized on the fifth day after injury, and was also detected in the regenerated skeletal muscle within 7-14 days after injury. Zhao et al., (2009) showed that morphometrically, the ratio of Caspase positive cells maximized at 5 days post-wounding in agree with our result.

Studies have demonstrated that Caspase proteases are also regulators of non-death functions, the most notable ones being those involving the maturation of a wide variety of cells such as red blood $\underline{\text { cells }}$ and skeletal muscle myoblasts ( Lamkanfi et al.,2007).

These results can be explained by Fernando et al.,(2002) who revealed that, Caspase- 3 activity has been linked with activation of the mitogen activated protein kinase (MAPK) through activation of intervening kinases. Therefore, this signal efficacy of the death effector protease also has a role of Caspase- 3 mediated signaling in skeletal muscle differentiation. Apoptosis and differentiation use the same signal cascade to engage MAPK activity. Indeed, such a contention bears significant merit when one considers that differentiating myoblasts share a remarkable similarity with many key cellular alterations endemic to apoptosis

In conclusion, investigation of time-dependent expression of Caspase- 3 in the peripheral area of contused rat skeletal muscle indicated that it may play a pivotal role in apoptosis of the wounded skeletal muscle and may be used as a marker for wound age determination, which would provide a new perspective for wound age estimation among the living instead of the histopathlogical changes. Further studies to estimate the expression of Caspase- 3 in other types of wounds in the living (e.g. cut wounds, contused...etc.) are recommended. 


\section{References}

Betz P, Nerlich A, Tübel J et al.,(1997): Detection of cell death in human skin wounds of various ages by an in situ end labeling of nuclear DNA fragments. Int. J. Legal. Med. 110:2403 .

Chazaud B, Brigitte M, Yacoub-Youssef $\mathrm{H}$ et al.,(2009): Dual and beneficial roles of macrophages during skeletal muscle regeneration. Exerc. Sport Sci. Rev. 37: 1822.

Dressler J, Bachmann L, Strejc P et al.,(2000): Expression of adhesion molecules in skin wounds: diagnostic value in legal medicine. Forensic Sci. Int. 113:173-176.

Fernando p, Kelly JF, Balazsi K et al.,(2002): Caspase 3 activity is required for skeletal muscle differentiation. Proc. Natl. Acad. Sci. U. S A. August 20; 99(17): 11025-11030.

Goldspink DF, Burniston JG, Ellison GM et al.,(2004): Catecholamine-induced apoptosis and necrosis in cardiac and skeletal myocytes of the rat in vivo: the same or separate death pathways? Exp. Physiol. 89: 407-416.

Grellner W, Georg T and Wilske J(2000): Quantitative analysis of proinflammatory cytokines (IL1beta, IL-6, TNF-alpha) in human skin wounds. Forensic Sci. Int. 113:251-264.

Guan DW, Ohshima $\mathrm{T}$ and Kondo $\mathrm{T}(2000)$ : Immunohistochemical study on Fas and Fas ligand in skin wound healing. Histochem. J. 32:85-91.

Hayashi T, Ishida Y, Kimura A et al.,(2004):Forensic application of VEGF expression to skin wound age determination. Int. J. Leg. Med. 118:320-325.

Huard J, Li Yand Fu FH(2002): Muscle injuries and repair: current trends in research. J. Bone Joint Surg. Am. 84:822-832.

Hurme T, Kalimo H, Lehto M et al.,(1991): Healing of skeletal muscle injury: an ultrastructural and immunohistochemical study. Med. Sci. Sports Exerc. 23:801-810.

Ishida Y, Kimura A, Takayasu T et al.,(2008):. Expression of oxygen-regulated protein 150 (ORP150) in skin wound healing and its application for wound age determination.Int. J. Legal. Med.122:409-414.

Jeruc J, Vizjak A, Rozman B et al.,(2006): Immunohistochemical expression of activated caspase- 3 as a marker of apoptosisin glomeruli of human lupus nephritis. Am. J. Kidney Dis. Sep;48(3):410-8.

Kagawa S, Matsuo A, Yagi Y et al.,(2009): The timecourse analysis of gene expression during wound healing in mouse skin. Leg. Med. 11:70-75.

Kondo T(2007): Timing of skin wounds. Leg. Med. 9: 109-114.

Kondo T, Ohshima Tand Eisenmenger W(1999) Immunohistochemical and morphometrical study on the temporal expression of interleukin-1alpha (IL-1alpha) in human skin wounds for forensic wound age determination. Int. J. Leg. Med .112:249-252.

Kondo T, Ohshima T, Mori R et al.,(2002): Immunohistochemical detection of chemokines in human skin wounds and its application to wound age determination. Int. J. Leg. Med. 116:87-91.

Lamkanfi M, Festjens N, Declercq W et al., (2007): Caspases in cell survival, proliferation and differentiation". Cell Death and Differentiation. 14 (1): 44-55.

Li M, Ding Y, Mu Y et al., (2011): Molecular cloning and characterization of caspase-3 in large yellow croaker (Pseudosciaena crocea). Fish Shell fish Immunol. Mar; 30(3):910-6.

McBrier NM, Lekan JM, Druhan LJ et al.,(2007): Therapeutic ultrasound decreases mechanogrowth factor messenger ribonucleic acid expression after muscle contusion injury. Arch. Phys. Med. Rehabil . 88:936-940.

McLennan IS (1996): Degenerating and regenerating skeletal muscles contain several subpopulations of macrophages with distinct spatial and temporal distributions. J. Anat.188:17-28.

Ortiz-Rey JA, Suarez-Penaranda JM, Munoz-Barus JI et al.,(2003):Expression of fibronectin and tenascin as a demonstration of vital reaction in rat skin and muscle. Int. J. Leg. Med. 117:356-360.

Ortmann C, Pfeiffer H and Brinkmann B(2000): A comparative study on the immunohistochemical detection of early myocardial damage. Int. J. Leg. Med. 113:215-220.

Papadimitriou JM, Robertson TA, Mitchell CA et al.,(1990): The process of new plasmalemma formation in focally injured skeletal muscle fibres. J. Struct. Biol.103:124-134.

Robertson TA, Maley MA, Grounds MD et al.,(1993): The role of macrophages in skeletal muscle regeneration with particular reference to chemotaxis. Exp. Cell Res. 207:321-33.

Sandri M, El Meslemani AH, Sandri C et al., (2001): Caspase 3 expression correlates with skeletal muscle apoptosis in Duchenne and facioscapulo human muscular dystrophy. A potential target for pharmacological treatment?J. Neuropathol. Exp. Neurol. Mar; 60(3):302-12.

Sato Y and Ohshima T (2000): The expression of mRNA of proinflammatory cytokines during skin wound healing in mice: a preliminary study for forensic wound age estimation (II). Int. J. Leg.Med. 113:140-145.

Srikuea R, Pholpramool C, Kitiyanant Y et al.,(2010): Satellite cell activity in muscle regeneration after contusion in rats. Clinical and Experimental Pharmacology and Physiology. 37: 1078-1086 
Stephenson T and Bialas Y (1996): Estimation of the age of bruising. Arch. Dis. Child. 74:53-55.

Sun JH, Wang YY, Zhang L etal.,(2010): Timedependent expression of skeletal muscle troponin I mRNA in the contused skeletal muscle of rats: a possible marker for wound age estimation. Int. J. Legal. Med. 124:27-33.

Takamiya M, Fujita S, Saigusa K et al.,(2008): Simultaneous detection of eight cytokines in human dermal wounds with a multiplex beadbased immunoassay for wound age estimation. Int. J. Legal. Med. 122: 143-148.

Takamiya M, Saigusa K, Kumagai R et al., (2005): Study's on mRNA expression of tissue-type plasminogen activator in bruises for wound age estimation. Int. J. Leg. Med. 119:16-21.

Takamiya M, Saigusa K, Nakayashiki N et al.,(2003): Studies on mRNA expression of basic fibroblast growth factor in wound healing for wound age determination. Int. J. Legal Med. 117:46-505.

Wang Q, Guan DW, Zhao R et al.,(2008):Timedependent expression of caspase-3 during contused skeletal muscle wound healing in rats. Fa Yi Xue Za Zhi. Feb; 24(1):5-7.
Wright-Carpenter T, Opolon P, Appell HJ et al.,(2004): Treatment of muscle injuries by local administration of autologous conditioned serum: animal experiments using a muscle contusion model. Int. J. Sports Med. 25:582587.

Yu TS, Cheng ZH, Li LQ et al., (2010): The cannabinoid receptor type 2 is timedependently expressed during skeletal muscle wound healing in rats. Int. J. Legal. Med. Sep; 124(5):397-404.

Zhang H, Zhu SH and Qin QS (2004): Immunohistochemical and morphometrical study on the expression of interleukin-10 (IL10) in different expressive parts during cutaneous wound healing in mice (in Chinese). J. Forensic Med.20:70-72.

Zhao R, Guan DW, Zhang W et al., (2009): Increased expressions and activations of apoptosisrelated factors in cell signaling during incised skin wound healing in mice: a preliminary study for forensic wound age estimation. Leg. Med.; 11:S155-S160.

\section{الملخص العربي

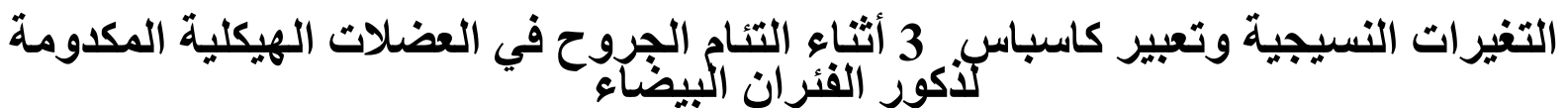

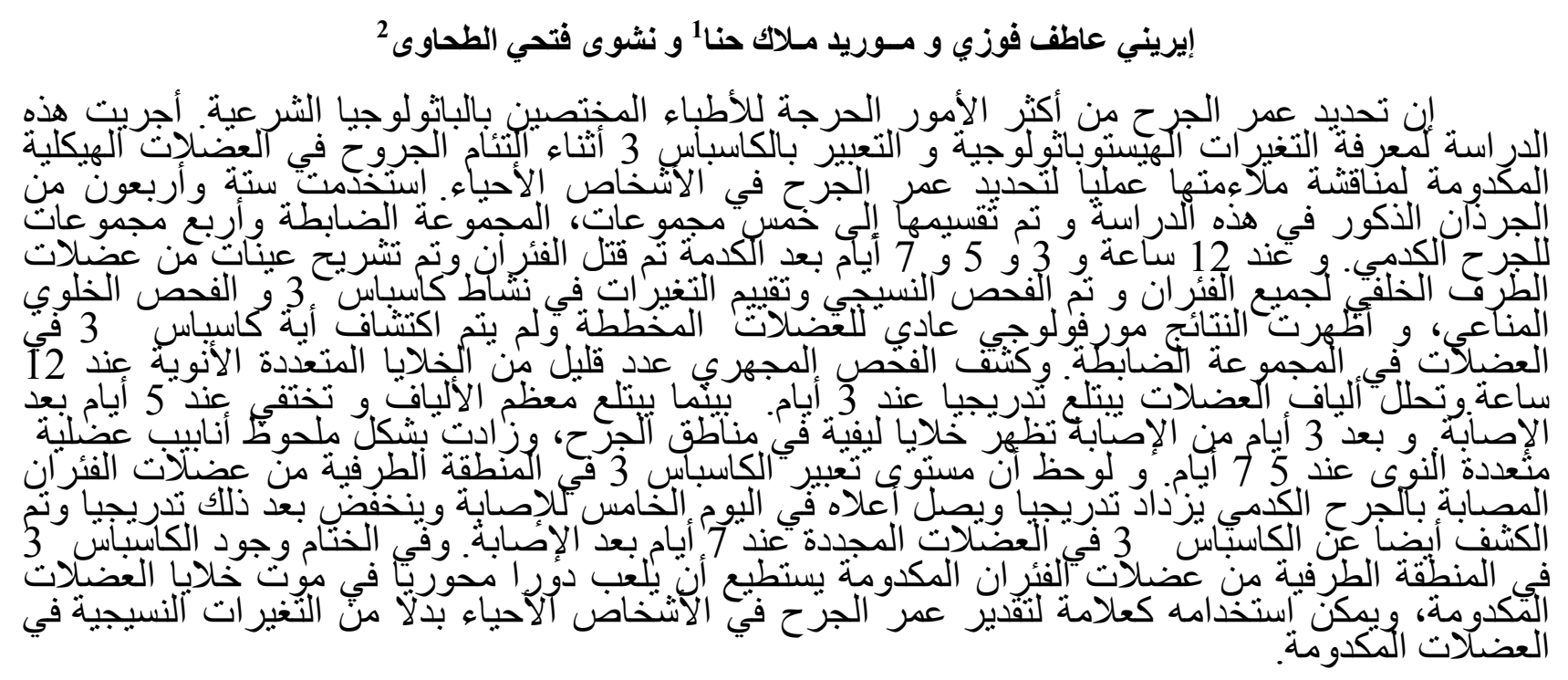

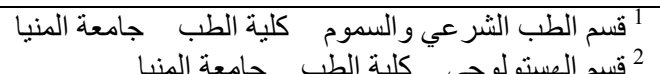

